Pesq. Vet. Bras. 37(7):681-685, julho 2017

DOI: $10.1590 /$ S0100-736X2017000700005

\title{
Intoxicação experimental por Niedenzuella stannea (Malpighiaceae) em ovinos ${ }^{1}$
}

\author{
Flávio H.B. Caldeiraa ${ }^{2,3 *}$, Felipe P. de Arruda ${ }^{2}$ Diego M. Shenkel ${ }^{2}$, Clairton \\ Marcolongo-Pereira ${ }^{4}$, Stephen T. Lee ${ }^{5}$, Daniel Cook ${ }^{5}$, Franklin Riet-Correa ${ }^{6}$ \\ e Edson Moleta Colodel ${ }^{7}$
}

\begin{abstract}
Caldeira F.H.B., Arruda F.P., Shenkel D.M., Marcolongo-Pereira C., Lee S.T., Cook D., Riet-Correa F. \& Colodel E.M. 2017. [Experimental poisoning by Niedenzuella stannea (Malpighiaceae) in sheep.] Intoxicação experimental por Niedenzuella stannea (Malpighiaceae) em ovinos. Pesquisa Veterinária Brasileira 37(7):681-685. Laboratório de Patologia Veterinária, Hospital Veterinário, Universidade Federal de Mato Grosso, Campus Universitário Cuiabá, Av. Fernando Corrêa da Costa 2367, Bairro Boa Esperança, Cuiabá, MT 78069-900, Brasil. E-mail: flaviobcaldeira@gmail.com

Niedenzuella stannea a sodium monofluoroacetate-containing plant cause sudden death in cattle in southern Mato Grosso State. This investigation describes the toxicity and clinical and pathological findings of experimental poisoning by $N$. stannea in sheep. Fruits, mature leaves and young leaves of the plant collected in properties near the margins of the Araguaia river basins with history of sudden death in cattle were used in the experiment. No clinical signs were observed in sheep ingesting doses between 10 and $40 \mathrm{~g} / \mathrm{kg}$ of mature leaves and $10 \mathrm{~g} / \mathrm{kg}$ of fruits. The animals had shown clinical signs when received young leaves of the plant orally in a single dose of $5,10,20 \mathrm{~g} / \mathrm{kg}$, and death occurred in sheep receiving $30 \mathrm{~g} / \mathrm{kg}$. The plant at the dose of $30 \mathrm{~g} / \mathrm{kg}$ of fresh leaves maintained its toxicity after being dried. The main clinical signs observed were anorexia, lethargy, dyspnea, tachycardia, and arrhythmia with a clinical course ranging 16-20 hours after the onset of clinical signs. In an hyperacute terminal phase the animals showed reluctance to move, frequent urination, engorged jugular, evident venous pulse, muscle tremor, sternal recumbence, lateral decubitus, and death. At necropsy engorgement of the atrium and large veins of the heart, and pulmonary edema were observed. Microscopically, the kidney had hydropic-vacuolar degeneration in the cytoplasm of the epithelial cells of the distal convoluted tubules. It is concluded that young leaves of $N$. stannea can cause intoxication in sheep and should be considered in the differential diagnosis of acute diseases or sudden death in sheep in regions where the plant exist.
\end{abstract}

INDEX TERMS: Poisonous plants, Niedenzuella stannea, plant poisoning, sudden death, sodium monofluoroacetate, sheep.

RESUMO.- Niedenzuella stannea é uma planta que contém monofluoracetato de sódio e é incriminada como causa de morte súbita em bovinos na Região Sul do Estado de Mato

\footnotetext{
${ }^{1}$ Recebido em 11 de agosto de 2016.

Aceito para publicação em 5 de dezembro de 2016.

${ }^{2}$ Programa de Pós-Graduação em Ciências Veterinárias (PPGVET), Faculdade de Medicina Veterinária (FAVET), Universidade Federal de Mato Grosso (UFMT), Campus Universitário, Av. Fernando Corrêa da Costa 2367, Bairro Boa Esperança, Cuiabá, MT 78069-900, Brasil.

${ }^{3}$ Instituto Federal de Educação, Ciência e Tecnologia de Rondônia (IFRO), Campus Colorado do Oeste, BR-435 Km 63, Zona Rural, Colorado do Oeste, R0, Brasil. *Autor para correspondência: flaviobcaldeira@ gmail.com
}

Grosso. Este estudo descreve a toxidez e achados clínicos e patológicos da intoxicação experimental por N. stannea em ovinos. Foram utilizados no experimento, frutos, fo-

\footnotetext{
${ }^{4}$ Faculdade de Medicina Veterinária, Centro Universitário Ritter dos Reis (UniRitter), Porto Alegre, RS, Brasil.

${ }^{5}$ Poisonous Plant Research Laboratory, Agricultural Research Service, U.S. Department of Agriculture, Logan, UT, United States.

${ }^{6}$ Laboratório de Anatomia Patológica, Hospital Veterinário, Centro de Saúde e Tecnologia Rural (CSTR). Universidade Federal de Campina Grande, Patos, PB 58700-310, Brasil.

${ }^{7}$ Faculdade de Medicina Veterinária (FAVET), UFMT, Campus Universitário, Av. Fernando Corrêa da Costa 2367, Bairro Boa Esperança, Cuiabá, MT 78069-900, Brasil.
} 
lhas maduras e folhas jovens de $N$. stannea coletadas em propriedades as margens de afluentes das bacias hidrográficas do Rio Araguaia onde havia histórico de morte súbita em bovinos. Folhas maduras em doses entre $10 \mathrm{e}$ $40 \mathrm{~g} / \mathrm{kg}$ e frutos na dose de $10 \mathrm{~g} / \mathrm{kg}$ não causaram alterações clínicas. Alterações clínicas foram observadas em ovinos que receberam a partir de $5 \mathrm{~g} / \mathrm{kg}$ de folhas jovens em dose única e a morte ocorreu nos que receberam a partir de $30 \mathrm{~g} / \mathrm{kg}$. A toxicidade na dose de $30 \mathrm{~g} / \mathrm{kg}$ se manteve após a secagem da planta. Os principais sinais clínicos foram anorexia, apatia, dispneia, arritmia e taquicardia em evolução clínica que variou de 16 às $20 \mathrm{~h}$. Notou-se em uma fase terminal hiperaguda, com evolução de 13 a 20 min., relutância ao movimento, micção frequente, jugular ingurgitada, pulso venoso evidente, tremor muscular, decúbito esternal e decúbito lateral e morte. Na necropsia os principais achados foram ingurgitamento de grandes veias, das aurículas do coração e edema pulmonar. Microscopicamente a principal alteração ocorreu no rim e caracterizou-se por degeneração hidrópico-vacuolar no citoplasma de epitélio de túbulos contorcidos distais. Conclui-se que $N$. stannea na fase de brotação é tóxica para ovinos e que a intoxicação por esta planta deve ser incluída no diagnóstico diferencial das doenças com curso clínico hiperagudo ou morte súbita em ovinos em regiões onde a planta existe.

TERMOS DE INDEXAÇÃO: Plantas tóxicas, Niedenzuella stannea, intoxicação por plantas, monofluoracetato de sódio, morte súbita, ovinos.

\section{INTRODUÇÃO}

Niedenzuella stannea (Griseb.) W.R. Anderson, planta pertencente à família Malpighiaceae, é encontrada no Brasil nas regiões Amazônica e do Pantanal, nos Estados do Pará, Mato Grosso e Mato Grosso do Sul (Mamede 2014). 0 gênero Niedenzuella é composto de plantas anteriormente classificadas nos gêneros Amorimia ou Tetrapterys (Malpighiaceae) por características morfológicas (Anderson 2006). Várias espécies dessas famílias são conhecidamente tóxicas para animais de produção (Tokarnia et al. 2012). As plantas do gênero Amorimia que causam intoxicações contêm monofluoroacetato de sódio e causam morte súbita associada ao exercício (Lee et al. 2012); enquanto que o consumo de Tetrapterys spp. causa fibrose cardíaca, aborto e espongiose do sistema nervoso central e seu princípio ativo é desconhecido (Tokarnia et al. 2012). Morte súbita é descrita como o evento em que "animais aparentemente sadios caem ao solo, em especial quando movimentados, e morrem em poucos minutos" (Tokarnia et al. 2012).

Niedenzuella stannea, que contém monofluoracetato de sódio (Arruda 2016), causa morte súbita associada ao exercício em bovinos no Estado de Mato Grosso, sendo que a intoxicação ocorre, com maior frequência, pelo consumo da brotação no período de seca (Caldeira et al. 2016). 0 objetivo deste trabalho é determinar a toxicidade e o quadro clínico-patológico induzido por $N$. stannea em diferentes fases de crescimento em ovinos.

\section{MATERIAL E MÉTODOS}

Para a reprodução experimental da doença foram utilizados 11 ovinos provenientes de propriedades com pastagens conhecidamente livres de $N$. stannea ou outra planta que cause morte súbita. Previamente os ovinos foram tratados com anti-helmíntico (doramectina $1 \%$ ) e passaram por exame clínico geral. Os ovinos foram mantidos em baias individuais com $2,16 \mathrm{~m}^{2}$, alimentados com silagem de milho e ração comercial com acesso ininterrupto à água.

Niedenzuella stannea (Grisebach) W.R. Anderson foi coletada próximo à margem de um de rios das bacias hidrográficas do Rio Araguaia, em uma propriedade com histórico de morte súbita em bovinos (S 16²18'58,6"; W 5256’02,1"). Uma amostra da espécie (Acesso \# 1514056, Michigan) foi coletada, prensada, seca, e enviada para o professor William R. Anderson, do herbário da Universidade de Michigan, Ann Arbor, Michigan, Estados Unidos, para identificação taxonômica. Dois estágios vegetativos de folhas de N. stannea foram coletados. Em agosto de 2013 coletaram-se folhas maduras, de arbustos de $N$. stannea, que se apresentavam fibrosas e com limbo verde-escuro (Fig.1), e em julho de 2014 foram coletadas folhas jovens, de brotações de N. stannea, que se apresentavam tenras e com limbo verde-claro (Fig.2). Foram, também, coletados frutos de N. stannea em junho de 2014.

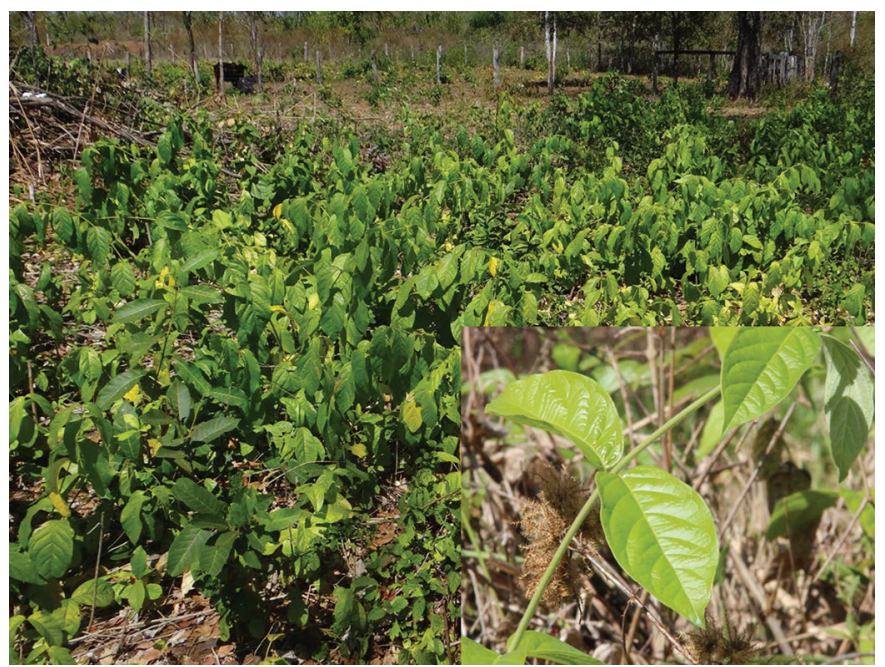

Fig.1. Niedenzuella stannea, julho/2014. Torixoréu, MT. Área de intensa brotação de N. stannea. Em detalhe, observam-se as folhas com limbo verde-claro.

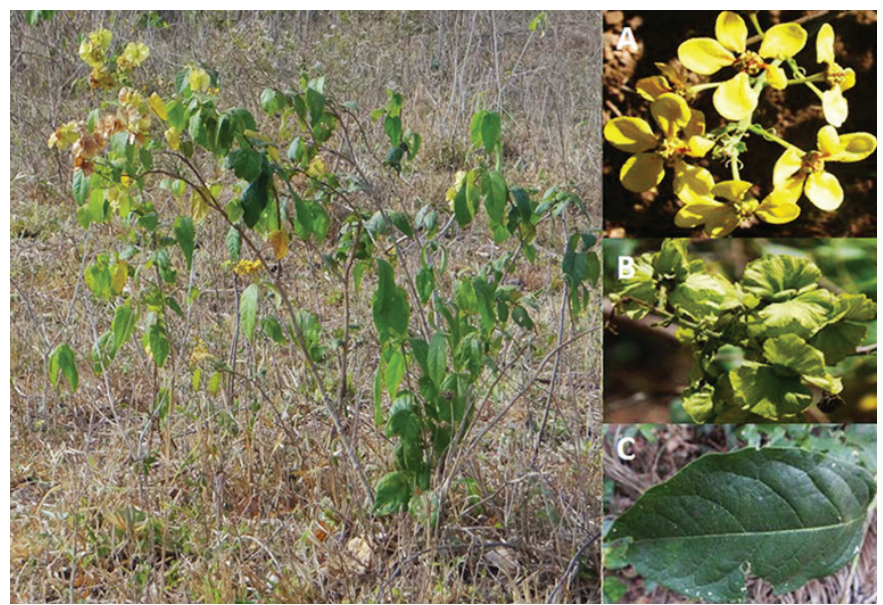

Fig.2. Arbusto de Niedenzuella stannea, agosto/2013. Torixoréu, MT. (A) Inflorescência. (B) Frutificação. (C) Folhas maduras fibrosas e com limbo verde-escuro. 

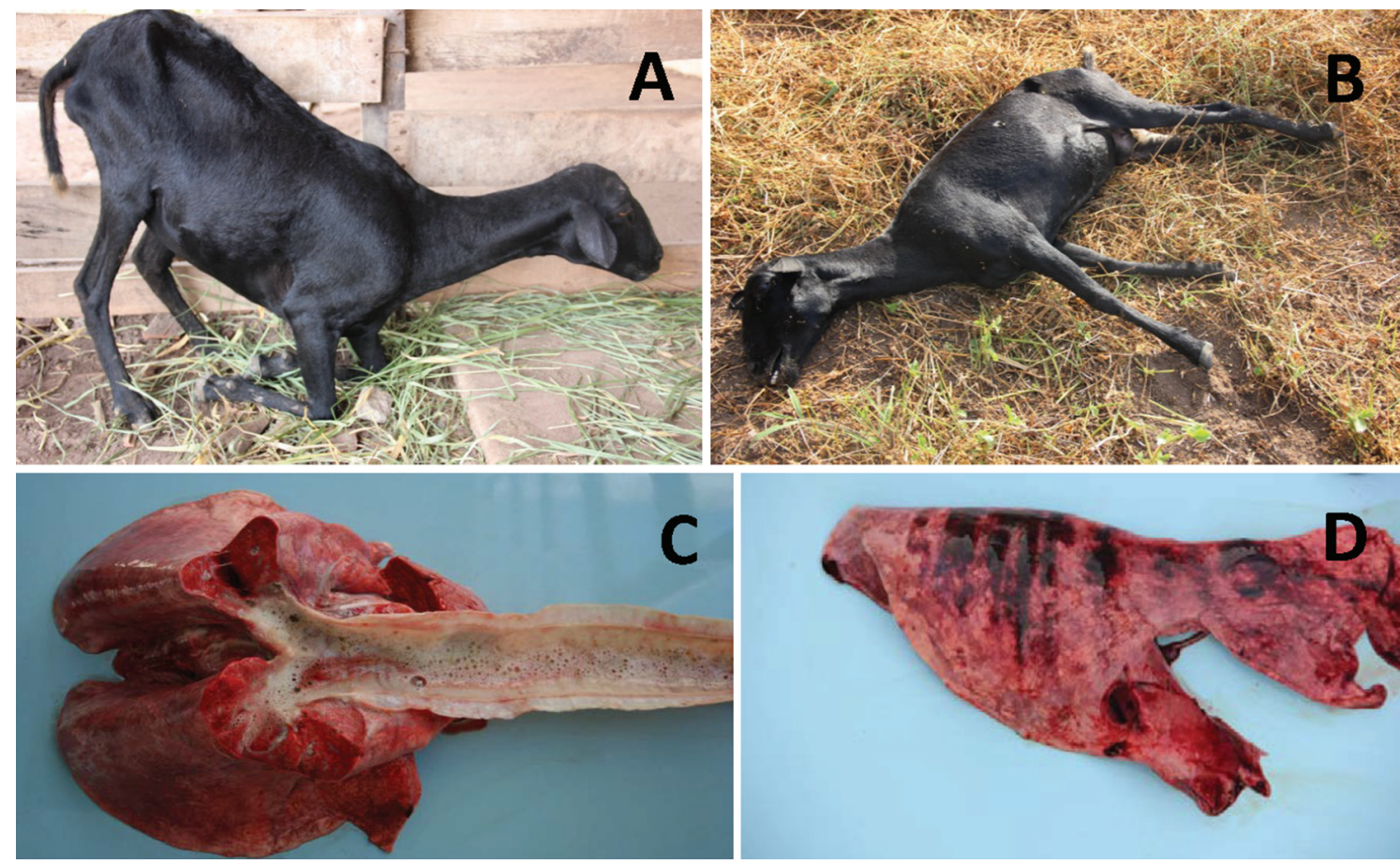

Fig.3. Intoxicação experimental por Niedenzuella stannea em ovinos. Ovino 4. (A) Relutância em se movimentar após 5 min de movimentação. (B) Decúbito lateral, após movimentação por 10 min, durante a evolução hiperaguda. (C) Grande quantidade de espuma brancacenta na traqueia e congestão difusa no pulmão. (D) Equimoses e sufusões na superfície do pulmão, acompanhando o padrão do gradil costal.

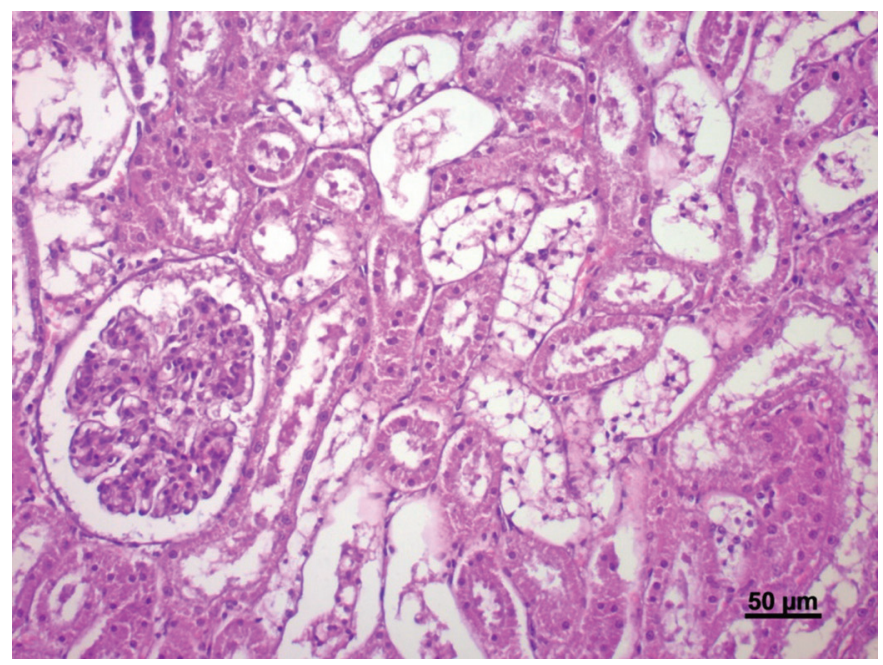

Fig.4. Intoxicação experimental por Niedenzuella stannea em ovinos. Ovino 4. Rim. Degeneração hidrópico-vacuolar dos túbulos uriníferos contorcidos distais associada à picnose nuclear. $\mathrm{HE}, \mathrm{Bar}=50 \mu \mathrm{m}$.

As doses administradas de N. stannea foram de 5, 10, 20 e $30 \mathrm{~g} / \mathrm{kg}$ de folhas jovens e 5, 10, 20 e $40 \mathrm{~g} / \mathrm{kg}$ de folhas maduras e $10 \mathrm{~g} / \mathrm{kg}$ de frutos. A administração foi por via oral. Para verificar a toxidez de folhas secas foi administrada a um ovino a dose de $30 \mathrm{~g} / \mathrm{kg}$ de folhas de $N$. stannea que foram pesadas frescas, e posteriormente dessecadas, em duas etapas. Primeiramente à sombra e à temperatura controlada de $14^{\circ} \mathrm{C}$ por 24 horas e depois em estufa de ventilação a temperatura de $55^{\circ} \mathrm{C}$ por mais 24 horas conforme protocolo utilizado por Schons et al. (2011). As doses foram calculadas com base no peso verde das folhas, posteriormente estas foram trituradas, misturada em $1 \mathrm{~L}$ de água e administrada por sonda esofágica. As doses e as repetições estão descritas no Quadro 1.

Aproximadamente cinco horas após a administração os ovinos eram movimentados por 10 minutos ou até apresentarem fadiga recusando-se a movimentar. Previamente e logo após o exercício aferiu-se as frequências respiratória e cardíaca dos ovinos. Os ovinos que se recuperaram ou que não apresentaram sinais clínicos tiveram acompanhamento com aferição dos parâmetros clínicos por pelo menos $24 \mathrm{~h}$ e acompanhamento visual por até sete dias. Os ovinos que morreram foram imediatamente necropsiados e fragmentos de todos os órgãos, incluindo o sistema nervoso central, foram fixados em formalina $10 \%$ e processados rotineiramente para análise histológica. 0 experimento foi realizado com aprovação do Comitê de Ética no Uso de Animais da Universidade Federal de Mato Grosso (protocolo N²3108016273/12-0).

\section{RESULTADOS}

Os achados clínicos relativos à administração de folhas e frutos de Niedenzuella stannea, estão apresentados no Quadro 1 . A dose de $10 \mathrm{~g} / \mathrm{kg}$ de frutos e doses únicas de 5,10 , 20 e 40g/kg de folhas maduras não causaram alteração clínica significativa nos ovinos deste experimento. Em relação administração de folhas jovens, a dose de $5 \mathrm{~g} / \mathrm{kg}$ (Ovino 1) causou sinais clínicos leves e recuperação clínica com aumento da frequência cardíaca (91bpm) notada logo após a movimentação, 5 horas após a administração de $N$. stannea. A dose de $30 \mathrm{~g} / \mathrm{kg}$ causou sinais clínicos acentuados e morte dos Ovinos 4, 5 e 6. A evolução clínica variou de 16 a 20 horas nos que consumiram folhas frescas (Ovinos 4 e 5), e $4 \mathrm{~h} 20 \mathrm{~min}$ para o que recebeu folhas dessecadas (Ovino 6). Os principais sinais clínicos observados foram anorexia, apatia, dispneia, taquicardia, arritmia, relutância ao movi- 
Quadro 1. Intoxicação experimental por Niedenzuella stannea (Malpighiaceae) em ovinos: delineamento experimental, quadro clínico, evolução e desfecho

\begin{tabular}{|c|c|c|c|c|c|c|c|c|c|c|c|}
\hline Ovino & $\begin{array}{l}\text { Conteúdo } \\
\text { administrado }\end{array}$ & $\begin{array}{l}\text { Dose } \\
(\mathrm{g} / \mathrm{kg})\end{array}$ & $\begin{array}{l}\text { Início sinais } \\
\text { clínicos }^{\mathrm{d}}\end{array}$ & $\begin{array}{l}\text { Evolução } \\
\text { clínica }\end{array}$ & $\begin{array}{c}\text { Duração da } \\
\text { fase superaguda }\end{array}$ & \multicolumn{3}{|c|}{$\begin{array}{c}\text { Frequência } \\
\text { Cardíaca (bpm) }\end{array}$} & $\begin{array}{l}\text { Frequência Respi- } \\
\text { ratória (mrpm) }\end{array}$ & $\begin{array}{l}\text { Desfecho } \\
\text { clínico }^{\mathrm{b}}\end{array}$ & $\begin{array}{l}\text { Sinais } \\
\text { clínicos }^{\mathrm{c}}\end{array}$ \\
\hline 1 & Folhas jovens & 5 & 11 horas & 5 horas & - & 88 & 91 & - & - & $\mathrm{R}$ & 5 \\
\hline 3 & Folhas jovens & 20 & - & - & - & 84 & - & - & - & NA & SA \\
\hline 4 & Folhas jovens & 30 & $6 \mathrm{~h} 07 \mathrm{~min}$ & $20 \mathrm{~h} 09 \mathrm{~min}$ & $13 \mathrm{~min}$ & 64 & 192 & 240 & 44 & M & $1-12$ \\
\hline 5 & Folhas jovens & 30 & $5 \mathrm{~h} 00 \mathrm{~min}$ & 16h20min & $20 \mathrm{~min}$ & 66 & 171 & 230 & 48 & M & $2-12$ \\
\hline 8 & Folhas maduras & 5 & - & - & - & 64 & - & - & - & NA & SA \\
\hline 9 & Folhas maduras & 10 & - & - & - & 88 & - & - & - & NA & SA \\
\hline 10 & Folhas maduras & 20 & - & - & - & 68 & - & - & - & NA & SA \\
\hline 11 & Folhas maduras & 40 & - & - & - & 66 & - & - & - & NA & SA \\
\hline
\end{tabular}

${ }^{\mathrm{a}}$ Frequência cardíaca após movimentação. T0 = antes da administração da planta, T1 $1=$ durante a evolução clínica, T2 = na evolução superaguda. ${ }^{\mathrm{b}} \mathrm{M}=$ morte, $\mathrm{R}=$ recuperação. 'Sinais clínicos apresentados, sendo: $1=$ anorexia, $2=$ apatia, $3=$ dispneia, $4=$ taquicardia, $5=$ arritmia, $6=$ relutância ao movimento, 7 = micção frequente, $8=$ jugular ingurgitada, $9=$ pulso venoso positivo, $10=$ tremor muscular, $11=$ decúbito esternal, $12=$ decúbito lateral. ${ }^{\mathrm{d}}$ Após administração de folhas e frutos de $N$. stannea. ${ }^{\mathrm{e}}$ Dose correspondente a $30 \mathrm{~g} / \mathrm{kg}$ de folha verde. ${ }^{\mathrm{f}} \mathrm{NA}=$ Não adoeceu. ${ }^{\mathrm{g}} \mathrm{SA}=\mathrm{Sem}$ alterações.

mento, micção frequente, jugular ingurgitada, evidenciação de pulso venoso e tremores musculares leves a moderado. Os ovinos manifestaram, ainda, uma fase hiperaguda, notada entre 13 e 20 minutos antes da morte e que se caracterizava por frequência cardíaca acima de $200 \mathrm{bpm}$, tremores musculares intensos, relutância à movimentação, dificuldade de se manter em estação, quedas ou decúbito esternal, decúbito lateral e morte.

As principais alterações macroscópicas observadas nos Ovinos 4, 5 e 6 foram ingurgitamento de aurículas e das veias cava cranial, das veias jugulares e das veias pulmonares. Nos pulmões havia equimoses e sufusões na superfície do órgão, acompanhando o padrão do gradil costal (Fig.3) nos Ovinos 4 e 5 . 0 órgão estava distendido contendo líquido seroso (edema), além de áreas multifocais com enfisema. A pleura visceral estava vermelho-escura e brilhosa. No coração havia equimoses epicárdicas no ventrículo esquerdo próximo ao sulco interventricular (Ovino 4). Observou-se também grande quantidade de espuma brancacenta na traqueia (Fig.3). Microscopicamente, havia no rim desses ovinos degeneração hidrópico-vacuolar dos túbulos contorcidos distais associada à picnose nuclear (Fig.4), com variação de intensidade, sendo considerada moderada no Ovino 4 e leve nos Ovinos 5 e 6 . Havia, ainda, edema e congestão difusa acentuada no pulmão em todos os ovinos e hemorragias focalmente extensas no pulmão e coração no Ovino 4. Nos demais órgãos não se observaram alterações microscópicas significativas.

\section{DISCUSSÃO E CONCLUSÃO}

Foi possível determinar que folhas jovens e frescas de $\mathrm{Nie}$ denzuella stannea causam a morte de ovinos, na dose de $30 \mathrm{~g} / \mathrm{Kg}$ e que a planta não perde a toxicidade após a secagem. Apesar de intoxicações espontâneas ainda não terem sido descritas em ovinos, $N$. stannea é uma importante causa de morte súbita associada ao exercício em bovinos no Estado de Mato Grosso (Caldeira et al. 2016). Assim, nas regiões Amazônicas e do Pantanal, a intoxicação por N. stannea deve ser considerada como diagnóstico diferencial da intoxicação por outras plantas que causam quadro clínico semelhante como Palicourea marcgravii (Tokarnia \& Döbereiner 1986), Amorimia pubiflora (Tokarnia \& Döbereiner 1973, Becker et al. 2013) e Amorimia amazonica (Schons et al. 2011).

O quadro de morte súbita em ovinos intoxicados experimentalmente por plantas como Palicourea marcgravii (Tokarnia \& Döbereiner 1986) e Amorimia pubiflora (Becker et al. 2013) estão relacionados a presença de monofluoracetato de sódio (MFA) nas plantas (Schultz et al. 1982). O MFA bloqueia competitivamente a aconitase e o ciclo de Krebs, o que reduz a produção de ATP, diminuindo o suprimento energético dos tecidos, principalmente do coração. Arruda (2016) detectou a presença de 0,003\% de MFA nas folhas jovens de $N$. stannea. A presença do MFA na planta provavelmente foi o responsável pelo desenvolvimento das alterações clínico-patológicas observadas. 0 quadro clínico foi reproduzido com administração de folhas coletadas de brotos de $N$. stannea na fase de desenvolvimento vegetativo da planta. Tem sido mencionado que a intoxicação em bovinos está associada ao consumo da planta em brotação, principalmente nos períodos de seca da região Centro-Oeste do Brasil ou correlacionado ao rebrote após a roça manual em pastagens infestadas por $N$. stannea (Caldeira et al. 2016).

Não foram observadas alterações clínicas e patológicas em ovinos que receberam folhas maduras de N. stannea nas doses de $5 \mathrm{a} 40 \mathrm{~g} / \mathrm{kg}$ e frutos na dose de $10 \mathrm{~g} / \mathrm{kg}$. Provavelmente, a ausência dos sinais clínicos está relacionada a não detecção de MFA nas folhas maduras da planta (Arruda 2016), evidenciando a importância da presença desse composto químico como causa do quadro clínico e patológico da intoxicação.

Os sinais clínicos observados em estudos experimentais com plantas da família Malpighiaceae que causam morte súbita são taquicardia e dificuldade respiratória e uma fase terminal, associada ao exercício. Nesta fase observa-se perda de equilíbrio, ataxia, tremores musculares, queda, decúbito lateral, movimentos de pedalagem, opistótono e morte em poucos minutos (Tokarnia \& Döbereiner 1973, 1986, Gava et al. 1998, Becker et al. 2013). Neste estudo 
todos os ovinos que receberam doses únicas de $30 \mathrm{~g} / \mathrm{kg}$ de $N$. stannea demonstraram sinais clínicos característicos de insuficiência cardíaca aguda e morte súbita, similares aos descritos em outros estudos experimentais com plantas que contém MFA (Tokarnia et al. 2012), incluindo os relatados nas intoxicações espontâneas por $N$. stannea em bovinos (Caldeira et al. 2016).

As alterações histológicas de degeneração hidrópico- vacuolar observadas nesse estudo são um achado frequente em animais intoxicados natural ou experimentalmente por plantas que contém MFA (Nogueira et al. 2010, Tokarnia et al. 2012). Nogueira et al. (2010) sugerem que existe uma relação entre o tempo de evolução dos sinais clínicos e a ocorrência da lesão renal. Em bovinos intoxicados com Palicourea marcgravii a intensidade da lesão renal foi maior quando o tempo decorrido entre a administração da planta e a morte era superior a 12 horas (Tokarnia \& Döbereiner 1986). Neste estudo a evolução clínica variou de $4 \mathrm{~h}$ e $20 \mathrm{~h}$, tempo suficiente para o estabelecimento da degeneração hidrópico vacuolar. Conclui-se que N. stannea na fase de brotação é tóxica para ovinos e que a intoxicação por esta planta deve ser incluída no diagnóstico diferencial das doenças com curso clínico hiperagudo em ovinos no Mato Grosso.

Agradecimentos.- Os autores agradecem ao Dr. William R. Anderson (University of Michigan Herbarium, Ann Arbor, Michigan, EUA) pela classificação botânica. À Fundação de Amparo à Pesquisa do Estado de Mato Grosso (FAPEMAT-Proc. no 156883/2014) ao INCT para o Controle das Intoxicações por Plantas/CNPq (Proc. no 573534/2008-0) pelo apoio Financeiro.

\section{REFERÊNCIAS}

Anderson W.R. 2006. Eight segregates from the neotropical genus Mascagnia (Malpighiaceae). Novon 16:168-204.
Arruda F.P. 2016. Dados não publicados (Universidade Federal de Mato Grosso, Cuiabá, MT).

Becker M., Caldeira F.H.B., Carneiro F.M., Oliveira L.P., Tokarnia C.H., Riet-Correa F., Lee S.T. \& Colodel E.M. 2013. Importância da intoxicação por Amorimia pubiflora (Malpighiaceae) em bovinos em Mato Grosso: reprodução experimental da intoxicação em ovinos e bovinos. Pesq. Vet. Bras. 33(9):1049-1056.

Caldeira F.B., Dias G.B., Arruda F.P., Lourenço F.M., Bezerra K.S., Riet-Correa F. \& Colodel E.M. 2016. Sudden Death associated with Niedenzuella stannea (Malpighiaceae) in cattle in the State of Mato Grosso, Brazil: importance and epidemiological aspects. Pesq. Vet. Bras. (Em publicação)

Gava A., Cristani J., Branco J.V., Neves D.S., Mondadori A.J. \& Souza R.S. 1998. Mortes súbitas em bovinos causadas pela ingestão de Mascagnia sp. (Malpiguiaceae) no Estado de Santa Catarina. Pesq. Vet. Bras. 18(1):16-20.

Lee S.T., Cook D., Riet-Correa F., Pfister J.A., Anderson W.R., Lima F.G. \& Gardner D.R. 2012. Detection of monofluoroacetato in Palicourea and Amorimia species. Toxicon 60:791-796.

Mamede M.C.H. 2014. Niedenzuella in: Lista de Espécies da Flora do Brasil. Jardim Botânico, Rio de Janeiro. Disponível em <http://reflora.jbrj.gov. br/jabot/floradobrasil/FB101449> Acesso em 15 Mai. 2014.

Nogueira V.A., França T.N., Peixoto T.C., Caldas S.A., Armién A.G. \& Peixoto P.V. 2010. Intoxicação experimental por monofluoroacetato de sódio em bovinos: aspectos clínicos e patológicos. Pesq. Vet. Bras. 30 (7):533-540.

Schons S.V., Mello T.L., Riet-Correa F. \& Schild A.L. 2011. Poisoning by Amorimia (Mascagnia) septium in sheep in northern Brazil. Toxicon 57:781-786.

Schultz R.A., Coetzer J.A.W., Kellerman T.S. \& Naudé T.W. 1982. Observations on the clinical, cardiac and histopathological effects of fluoracetate in sheep. Onderstepoort J. Vet. Res. 49:237-245.

Tokarnia C.H. \& Döbereiner J. 1973. Intoxicação por Mascagnia pubiflora em bovinos no Estado do Mato Grosso. Pesq. Agrop. Bras. 8:61-68.

Tokarnia C.H. \& Döbereiner J. 1986. Intoxicação por Palicourea marcgravii (Rubiaceae) em bovinos no Brasil. Pesq. Vet. Bras. 6:73-92.

Tokarnia C.H., Brito M.F., Barbosa J.D., Peixoto P.V. \& Döbereiner J. 2012. Plantas Tóxicas do Brasil para Animais de Produção. $2^{\underline{a}}$ ed. Editora Helianthus, Rio de Janeiro. 